\title{
Diet palatability and amphetamine-induced anorexia
}

\author{
PAUL J. WELLMAN and DAVID J. PITTENGER \\ Texas A\&M University, College Station
}

and

\author{
KENNETH C. WIKLER \\ Bucknell University, Lewisburg, Pennsylvania
}

\begin{abstract}
The influence of diet palatability and caloric density on amphetamine anorexia was examined in two experiments. In Experiment 1, rats were offered a palatable but hypercaloric high-fat diet, a chow diet, a pellet diet, or a palatable mineral-oil diet equal in caloric density to the chow and pellet diets. Although the rats displayed differential acceptance of the diets during saline intake tests, identical percent changes in intake with amphetamine treatment $(1.0,2.0$, and $4.0 \mathrm{mg} / \mathrm{kg})$ were observed. In a second experiment, rats were offered the chow and mineral-oil diets in a preference sequence before and after intake testing to ensure that these diets were indeed different in palatability. Although the rats displayed a considerable preference for the mineral-oil diet over the chow diet, comparable percent changes in diet intake with amphetamine $(.25, .50,1.0$, and $2.0 \mathrm{mg} / \mathrm{kg}$ ) were observed. These data, therefore, did not support the notion that enhancement of diet palatability may offset the anorexic property of amphetamine but did support a malaise interpretation of amphetamine anorexia.
\end{abstract}

Of the numerous central nervous system stimulants ascribed anorexic properties over the last four decades, amphetamine represents the prototypic anorexic agent. Although it has been suggested that amphetamine induces anorexia via a CNS satiety mechanism, presumably within the lateral hypothalamus or dorsolateral tegmentum (Ahlskog \& Hoebel, 1973; Leibowitz, 1975), more recent observations have questioned the specificity and mechanism of action of amphetamine anorexia. That the anorexia induced by amphetamine is not specific to food intake is suggested by a report from Carey and Goodall (1974), who observed that amphetamine induced dose-dependent suppression of water intake. Moreover, it is unclear that the anorexia induced by amphetamine results from a reduction of food motivation, since amphetamine induces conditioned taste aversion at dosages that produce anorexia for both food and water intake (Berger, 1972; Carey, 1973; Carey \& Goodall, 1974). That the aversive property of amphetamine may contribute to its anorexic property is further supported by the observations that lesions of the dorsolateral tegmentum attenuate both the anorexia and the conditioned taste aversion in-

Correspondence may be addressed to P. J. Wellman, Department of Psychology, Texas A\&M University, College Station, Texas 77843.

-Article accepted by previous editor, Richard F. Thompson. duced by amphetamine (Wellman, McIntosh, \& Guidi, 1981; Wellman \& Peters, 1980). Although it is likely that at least a portion of the anorexia induced by amphetamine may result from malaise, the precise nature (e.g., hyperthermia, Carey, 1979; arousal, Carlton, 1963) of this effect is unclear.

Thus, although amphetamine suppresses food intake, the relationship between this effect and the regulation of food motivation is currently speculative. Previous investigations of amphetamine anorexia have largely concentrated on the neuroanatomical and neurochemical basis of amphetamine anorexia, with few experiments examining amphetamine anorexia in the context of manipulations known to influence food motivation. Cole (1979), for example, noted that manipulation of food deprivation produced significant changes in amphetamine anorexia, with increased food motivation interacting with the magnitude of anorexia. Wellman and Peters (1980) reported an interaction between the magnitude of amphetamine anorexia and the palatability of the test diet. Specifically, rats displayed minimal anorexia when offered a palatable high-fat test diet, yet displayed substantial anorexia to the same amphetamine dosages when offered a less palatable pellet test diet. Their findings are similar to earlier outcomes in which the magnitude of anorexia observed to a variety of anorexic agents (e.g., cholecystokinin, intraduodenal glucose, hepatic-portal infusions of adrenaline; Campbell \& Davis, 1974; Krinsky, Lotter, 
\& Woods, 1979; Russek, 1971) varied with diet palatability. These data suggest that enhancement of diet palatability may be sufficient to offset the anorexic property of a variety of treatments.

A firm conclusion regarding an interaction between diet palatability and anorexia in general and amphetamine anorexia in particular, however, awaits the demonstration that these effects result from differences in diet palatability and not from differences in the caloric density of the test diets. In the Krinsky et al. (1979) and Wellman and Peters (1980) experiments, the high-fat test diets contained nearly twice the calories per gram of the pellet test diet; thus, although amphetamine or CCK produced differential anorexia in terms of grams of food consumed, each drug may have produced equivalent suppressions of caloric intake. Experiment 1 further explored the potential interaction between diet palatability and amphetamine anorexia using ground chow, pellet, mineral-oil, and high-fat test diets. These diets have been shown to induce differential overeating in ventromedial hypothalamic lesioned rats, presumably because of differential palatability (Corbit \& Stellar, 1964). The mineral-oil test diet was used as a control for the calorically dense high-fat test diet because this diet, although greasy, is equicaloric with the ground-chow and pellet test diets.

\section{EXPERIMENT 1}

\section{Method}

Animals. The animals were 32 female Long-Evans hooded rats (Blue Spruce Farms, Inc.), 60 days old and weighing 185-232 g at the beginning of the experiment. The rats were individually housed in standard wire-mesh cages in a temperature-controlled $\left(23^{\circ} \mathrm{C}\right)$ colony room under continuous illumination. They were provided with food (described below) and tap water, except as given in the schedules below, throughout the experiment.

Drugs. A $.9 \%$ saline solution was prepared by dissolving sodium chloride (Fischer) into sterile distilled water. Amphetamine solutions $(1.0,2.0$, and $4.0 \mathrm{mg} / \mathrm{ml})$ were prepared by dissolving dextroamphetamine sulfate (Sigma Chemical Company) into sterile distilled water. All solution concentrations were calculated as the salt (Seiden \& Dykstra, 1977).

Diets. Four test diets were used in this experiment. A groundchow (CHOW) diet and a pellet (PELL) diet were obtained commercially (Purina Rat \& Mouse Diet; $3.6 \mathrm{kcal} / \mathrm{g}$ ). A high-fat (HIFT) diet consisted of 2 parts Purina Chow and 1 part melted vegetable oil (Crisco, $5.6 \mathrm{kcal} / \mathrm{g}$ ), while a mineral-oil high-fat (MOHF) diet containing $3.6 \mathrm{kcal} / \mathrm{g}$ consisted of $66 \%$ Purina Chow, $14.9 \%$ Crisco, and $18.1 \%$ mineral oil (w/w; Corbit \& Stellar, 1964). The HIFT and MOHF diets were prepared fresh prior to each intake test, and all diets were presented to the rats in glass jars.

Procedure. The rats were assigned randomly to one of the four diet conditions ( $N=8$ each) and were trained to consume the respective test diet on 7 consecutive days. For these tests, the rats were deprived of food, but not water, for $23 \mathrm{~h}$ and were given access to the test diet, but not water, for $60 \mathrm{~min}$ in a plastic holding cage. Food intakes, corrected for spillage, were recorded to the nearest $.1 \mathrm{~g}$.

A drug sequence, using the procedures described above, was administered using the following injection regimen: saline-salinesaline-drug-saline-drug-saline-drug-saline. All injections were given in a volume of $1.0 \mathrm{ml} / \mathrm{kg}$ (ip) $30 \mathrm{~min}$ prior to each intake test. The order of drug injections was randomized for each diet group, with each rat receiving every drug dosage once. Saline injections were given prior to and between drug treatments to ensure that baseline diet intake remained stable.

Statistical analyses. The intake data from the drug sequence were analyzed as a mixed factorial design with diet (CHOW, PELL, MOHF, and HIFT) and drug dosage $(0.0,1.0,2.0$, and $4.0 \mathrm{mg} / \mathrm{kg}$ ) as between and repeated factors, respectively. Subsequent comparisons of means were made at the .05 level of significance using two-tailed a posteriori Tukey tests (Kirk, 1968).

\section{Results}

Figure 1 presents the changes in food intake (left panel) and the percent changes in food intake (right panel) produced by the amphetamine treatments of Experiment 1. Analyses of the food intake data revealed significant main effects of diet $[F(3,28)=4.7$, $\mathrm{p}<.01]$ and drug dosage $[\mathrm{F}(3,84)=8.8, \mathrm{p}<.001]$ but not of the interactions between these factors. As can be seen in the figure, saline-treated rats displayed differential acceptance of the various diets, with rats offered the MOHF test diet eating significantly more food than the rats offered the HIFT, PELL, and CHOW test diets $[t(14) \geqslant 2.3$, for each MOHF-diet comparison, $\mathrm{p}<.05$ ]. Amphetamine treatment produced significant, dose-dependent suppressions of food intake [saline vs. drug, $t(14) \geqslant 2.3$ for each comparison, $\mathrm{p}<.05$ ], regardless of test diet. Thus, although these diets were clearly different in palatability, as indexed by acceptance in these brief tests, amphetamine treatment induced comparable suppressions of food intake. This conclusion was strengthened by an analysis of the percent change scores (Figure 1, right panel) revealing a significant effect of drug $[F(2,56)=7.5, p<.001]$ but not of diet or of the interaction between drug and diet.

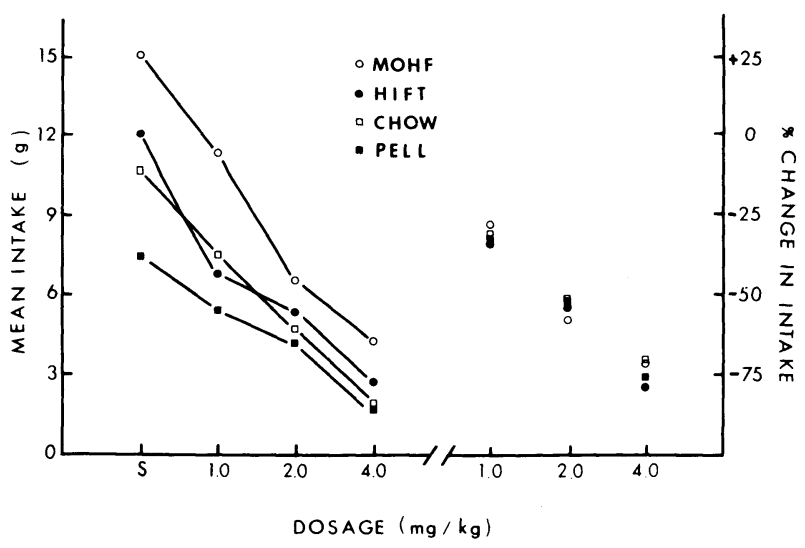

Figure 1. The left panel presents group mean food intake after treatment with saline(s) and with $1.0,2.0$, and $4.0 \mathrm{mg} / \mathrm{kg}$ damphetamine sulfate, and the right panel presents group mean percent change in intake (1.0 - drug intake/saline intake) induced by each drug dosage. 


\section{Discussion}

The outcomes of Experiment 1 did not demonstrate the expected interaction between diet palatability and the magnitude of amphetamine anorexia. Although rats offered diets classically considered to possess different magnitudes of palatability displayed different degrees of diet acceptance, amphetamine produced equivalent suppressions of intake, regardless of test diet. Acceptance of a food substance or liquid is, however, at best a poor indicator of diet palatability (Falk, 1971; Miller, 1957) relative to a preference paradigm. To ensure that the diets were, indeed, discriminably more or less palatable, a second experiment was carried out, using the CHOW and MOHF diets, in which the influence of amphetamine on diet intake was assessed between diet preference tests.

\section{EXPERIMENT 2}

\section{Method}

Animals. The animals were 13 female Long-Evans hooded rats (Blue Spruce Farms, Inc.), 60 days old and weighing 196$244 \mathrm{~g}$ at the beginning of the experiment. The rats were individually housed in standard plastic cages (Lab Co.), containing pine shavings, in a temperature-controlled $\left(23^{\circ} \mathrm{C}\right)$ colony room under continuous illumination.

Diets. Two test diets, slightly different in caloric density from those of Experiment 1, were used in this experiment. A finely ground-chow (CHOW) diet containing $4.06 \mathrm{kcal} / \mathrm{g}$ was prepared by milling a standard pellet chow diet (Wayne). An equicaloric, but greasy, mineral oil and high-fat (MOHF) diet consisted of $66 \%$ Wayne ground-chow, $14.9 \%$ melted vegetable oil, and $18.1 \%$ mineral oil $(w / w)$.

Procedure. The rats were exposed to the $\mathrm{CHOW}$ and MOHF test diets on 4 consecutive days in a preference test sequence. The rats were deprived of food, but not water, for $23 \mathrm{~h}$ prior to each test and were given $60 \mathrm{~min}$ access to both diets in separate intake cages. The diets were presented to the rats in glass jars secured upright by cardboard inserts placed at one end of each cage. Diet position was alternated over test days to prevent position preferences, and diet intakes (corrected for spillage) were recorded to the nearest $.1 \mathrm{~g}$.

After determining that the MOHF test diet was indeed preferred to the CHOW test diet, the influence of amphetamine $(.25, .50$, 1.0 , and $2.0 \mathrm{mg} / \mathrm{kg}$ ) on diet intake was assessed in separate diet groups. The drug sequence consisted of saline-saline-saline-salinesaline-drug-saline-drug-saline-drug-saline-drug. For the first group $(\mathrm{N}=6)$, the CHOW diet was used, and for the second group $(\mathrm{N}=7)$, the MOHF test diet was used. Saline or drug injections (ip), in a volume of $1.0 \mathrm{ml} / \mathrm{kg}$, always preceded the 60 -min test period by $30 \mathrm{~min}$. Each rat received every amphetamine dosage once per diet sequence with order randomized within each diet sequence. To ensure that drug treatment did not alter the baseline preference of the rats for the MOHF test diet, an additional 4-day preference test sequence, identical to that described above, was carried out after the drug sequence.

Statistical analyses. The CHOW and MOHF intakes from each preference test were converted to preference scores (MOHF intake/ MOHF + CHOW intakes), and these data were subjected to a one-way ANOVA (Kirk, 1968). The intake data from the drug sequence were analyzed as a mixed factorial design with diet (MOHF, CHOW) and drug dosage $(0.0, .25, .5,1.0$, and 2.0 $\mathrm{mg} / \mathrm{kg}$ amphetamine) as between and repeated factors, respectively.
Table 1

Mean Group Preference Scores (MOHF Intake/Total Intake) for CHOW and MOHF Rats Before and After the Amphetamine Test Sequence

\begin{tabular}{ccccccc}
\hline & & \multicolumn{2}{c}{$\begin{array}{c}\text { Before Drug } \\
\text { Treatment }\end{array}$} & & \multicolumn{2}{c}{$\begin{array}{c}\text { After Drug } \\
\text { Treatment }\end{array}$} \\
Diet & $\mathrm{N}$ & Mean & SEM & & Mean & SEM \\
\hline CHOW & 6 & .94 & .04 & .94 & .09 \\
MOHF & 7 & .93 & .08 & .94 & .08 \\
\hline
\end{tabular}

\section{Results and Discussion}

Table 1 presents mean group preference scores (MOHF intake/total intake), collapsed across each preference sequence, for the preference sequence before and after the amphetamine test sequence. As is clear from inspection of these data, the rats displayed a considerable preference for the MOHF diet before and after the amphetamine test sequence with no differences either between the preference sequences or between diet groups within a sequence. The preference data therefore confirm the acceptance data of Experiment 1 and strengthen the argument that these differences in preference reflect differences in the palatability (e.g., texture, greasiness, etc.) of the MOHF diet relative to the $\mathrm{CHOW}$ diet rather than any differences in caloric content or postingestional consequences.

The influence of amphetamine $(.25, .5,1.0$, and $2.0 \mathrm{mg} / \mathrm{kg}$ ) on the intake of either a CHOW or a MOHF diet is presented in Figure 2. Group mean intakes are depicted in the left panel, and percent changes in intake (1.0-drug intake/saline intake) are presented in the right panel.

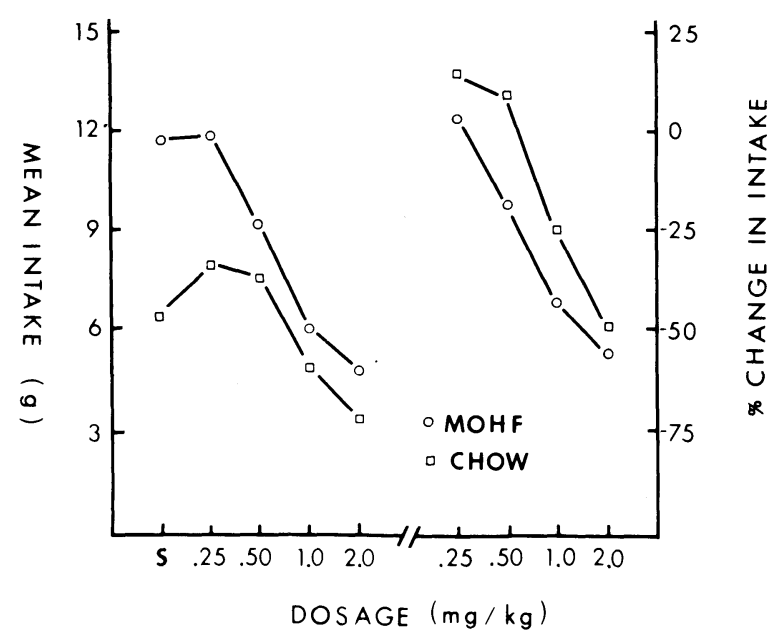

Figure 2. Mean group intakes (left panel) and percent change in intake (right panel) induced by amphetamine $(.25, .5,1.0$, and $2.0 \mathrm{mg} / \mathrm{kg}$ ) in rats fed either a chow diet (CHOW) or a more palatable but equicaloric mineral-oil high-fat diet (MOHF). 
Analysis of variance of the raw diet intakes after saline and amphetamine treatment revealed significant effects of diet $[F(1,11)=15.2, p<.00001]$ and drug $[F(4,44)=21.9, p<.00001]$, but the interaction between these factors was not significant. Subsequent comparisons revealed that rats offered the MOHF test diet ate significantly more food after saline treatment than did rats offered the groundchow test diet $[\mathrm{t}(11)=2.4, \mathrm{p}<.05]$. For both diets, amphetamine produced nonsignificant increases in intakes at low dosages, but produced dose-dependent and significant suppression of intake at 1.0 and $2.0 \mathrm{mg} / \mathrm{kg}[\mathrm{t}(11)=\geqslant 2.5, \mathrm{p}<.05$, for each drugsaline comparison], with no differences between diets. These outcomes were confirmed by a second analysis of variance, using percent change in intake as the dependent measures. Again, there were observed significant main effects of diet $[F(1,11)=27.4, p<.0003]$ and drug $[\mathrm{F}(3,33)=28.7, \mathrm{p}<.00001]$ and a nonsignificant interaction between diet and drug.

As noted above, rats offered the CHOW diet consumed significantly less of that diet after saline treatment before and between drug tests. As a consequence, although both groups displayed comparable mean group body weights at the outset of intake testing, the body weights of the CHOW group declined and at the end of the amphetamine test sequence were maintained at a level $88 \%$ of that maintained by rats fed the MOHF test diet. Because body-weight differences per se mask or produce between-group differences in food motivation (Beatty, 1978; Jacobs \& Sharma, 1967) and taste reactivity, a further analysis of the percent change data was performed using average body weight for each animal as a covariate. This analysis, however, did not reveal a significant effect of diet or of the interaction between diet and drug.

\section{DISCUSSION}

The purpose of the present experiments was to further elucidate the influence of diet palatability on amphetamine anorexia. Although several instances of an interaction between diet and palatability and anorexia have been observed (Campbell \& Davis, 1974; Krinsky, Lotter, \& Woods, 1979; Russek, 1971; Wellman \& Peters, 1980), the present experiments failed to reveal any influence of diet on the magnitude of anorexia induced by a wide range of amphetamine dosages. Although rats offered various diets displayed differential acceptance of these diets (Experiment 1) and although rats offered mineral-oil and equicaloric chow diets displayed differential preference for these diets (Experiment 2), amphetamine induced similar changes in food intake, regardless of test diet.

Given the dramatic interaction between diet and anorexia observed in the Wellman and Peters (1980) paper, the failure to observe such an interaction in either of the present experiments was unexpected. Consideration of several procedural variables that may have resulted in an artifactual interaction between diet and anorexia, however, may serve to explain the former outcomes. In the Wellman and Peters (1980) experiment, the rats were reduced to, and maintained at, $80 \%$ body weight throughout the drug tests. Moreover, the rats were offered the palatable high-fat diet in the first drug sequence, followed by the less palatable pellet diet in the second drug sequence. Jacobs and Sharma (1967) argue that food deprivation may enhance affective responsivity to palatable solutions; thus, maintaining rats at $\mathbf{8 0 \%}$ body weight may have altered the affective response of these animals to the high-fat diet in a fashion not apparent in rats in the present experiments tested at $100 \%$ body weight. Moreover, the sequence of testing used in the Wellman and Peters (1980) experiment may have resulted in a negative contrast effect (Crespi, 1942), such that rats tested with a palatable diet followed by a less palatable diet display an enhanced anorexic response during the latter drug sequence. Thus, conducting anorexic tests in rats maintained at $80 \%$ body weight and successfully offered palatable and less palatable test diets may have artifactually created an interaction between diet and amphetamine anorexia. The influence of body weight level was also apparent in the second experiment, in which rats fed the CHOW and MOHF diets displayed gradual divergence of body weight over the course of intake testing. The influence of differential body weight was clearly observed in that, when no account was made of body weight, amphetamine seemingly produced greater percent changes in the CHOW group relative to the changes in food intake displayed by the MOHF diet group whereas no significant effect of diet was apparent between the anorexic responses of these diet groups when body weight was used as a covariate for the percent change analyses.

Finally, the present data suggest that the anorexia induced by amphetamine may be best accounted for in terms of a malaise mechanism rather than a CNS satiety mechanism. Were amphetamine to act via a satiety process that precisely regulates the number of calories consumed by an animal, an interaction between amount eaten and amphetamine dosage might be expected. More specifically, amphetamine ought to induce comparable suppression of caloric intake but differential suppression in terms of amount consumed when caloric density (and presumably palatability) is varied. On the other hand, were amphetamine to act via a simple malaise (or competing responses) process, one might logically expect amphetamine to induce comparable suppression of amount of food consumed and differential suppression of caloric intake over a range of caloric densities. The present experiments, in which comparable suppressions of amount consumed but not of caloric 
intake were observed, seem therefore to support the notion that amphetamine produces anorexia via activation of a nonspecific malaise process (Carey \& Goodall, 1974; Wellman, McIntosh, \& Guidi, 1981; Wellman \& Peters, 1980).

\section{REFERENCES}

Ahlskog, J. E., \& Hoebel, B. G. Overeating and obesity from damage to a noradrenergic system in the brain. Science, 1973, 182, 166-168.

BEATTY, W. W. Operant responding in rats with dietary obesity: A systematic replication. Physiology \& Behavior, 1978, 21, 671-672.

BERGER, B. D. Conditioning of food aversions by injections of psychoactive drugs. Journal of Comparative and Physiological Psychology, 1972, 81, 21-26.

Campbell, C. S., \& Davis, J. D. Peripheral control of food intake: Interaction between test diet and postingestive chemoreception. Physiology \& Behavior, 1974, 12, 377-384.

CAREY, R. J. Long-term aversion to a saccharin solution induced by repeated amphetamine injections. Pharmacology, Biochemistry and Behavior, 1973, 1, 265-270.

CAREY, R. J. Facilitation of responding for rewarding brain stimulation by a high dose of amphetamine when hyperthermia is prevented. Psychopharmacology, 1979, 61, 267-271.

CAREY, R. J., \& Goodall, E. B. Amphetamine-induced aversion: A comparison of d- versus l-amphetamine. Pharmacology, Biochemistry and Behavior, 1974, 2, 325-330.

Carlton, P. L. Cholinergic mechanisms in the control of behavior. Psychological Review, 1963, 70, 19-39.

Cole, S. O. Interaction of food deprivation with different measures of amphetamine effects. Pharmacology, Biochemistry and Behavior, 1979, 10, 235-238.

Corbit, J. D., \& Stellar, E. Palatability, food intake and obesity in normal and hyperphagic rats. Journal of Comparative and Physiological Psychology, 1964, 58, 63-67.
Crespi, L. P. Quantitative variation of incentive and performance in the white rat. American Journal of Psychology, 1942, 55, 467-517.

FALK, J. L. Determining changes in vital functions: Ingestion. In R. D. Myers (Ed.), Methods in psychobiology (Vol. 1). New York: Academic Press, 1971.

Jacobs, H. L., \& Sharma, K. N. Taste versus calories: Sensory and metabolic signals in the control of food intake. Annals of the New York Academy of Sciences, 1967, 157 (R2), 1084-1125.

KIRK, R. E. Experimental design: Procedures for the behavioral sciences. Belmont, Calif: Brooks/Cole, 1968.

Krinsky, R., LotTer, E. C., \& Woods, S. C. Appetite suppression caused by CCK is diet specific in VMH-lesioned rats. Physiological Psychology, 1979, 7, 67-69.

LEIBOWITZ, S. F. Catecholaminergic adrenergic mechanisms of the lateral hypothalamus: Their role in the mediation of amphetamine anorexia. Brain Research, 1975, 98, 529-545.

Miller, N. E. Experiments on motivation. Science, 1957, 126, 1271-1278.

RUSSEK, M. Hepatic receptors and the neurophysiological mechanisms controlling feeding behavior. In S. Ehrenpreis (Ed.), Neuroscience research (Vol. 4). New York: Academic Press, 1971.

Seiden, L. S., \& Dykstra, L. A. Psychopharmacology: A biochemical and behavioral approach. New York: Van Nostrand, 1977.

Wellman, P. J., McIntosh, P., \& Guidi, E. Effects of dorsolateral tegmental lesions on amphetamine- and lithium-induced taste aversion. Physiology \& Behavior, 1981, 26, 341-344.

Wellman, P. J., \& Peters, R. H. Effects of amphetamine and phenylpropanolamine on food intake in rats with ventromedial hypothalamic or dorsolateral tegmental damage. Physiology \& Behavior, 1980, 25, 819-827.

(Manuscript received June 30, 1981; accepted for publication November 5,1981 .) 